\title{
A Meta-Analysis Summary of Information Technology Lack of Connectivity and Usability in Patient Centered Care
}

\author{
Enid Nwosisi, Linda Carl, and Christopher Nwosisi, Senior Member, IACSIT
}

\begin{abstract}
In the past 30 years, information technology (IT) has become commonplace in the healthcare arena. IT provides beneficial attributes in easing the documentation processes in healthcare while creating a host of new documentation challenges. Usability is one problem of bedside computer charting that takes the nurse's attention away from direct patient care time. The problem addressed in this proposal is that IT documentation does not always provide precision upon reviewing the patient care information. For example, a system that is not specialty specific may not ask accurate questions to get targeted information to render optimal patient care. The purpose of this research study is to analyze IT connectivity and usability using qualitative meta-analysis summary by reviewing research that speaks to solving the challenges.
\end{abstract}

Index Terms-Information technology (IT), connectivity, usability, patient centered care.

\section{INTRODUCTION}

Information technology (IT) has become commonplace in healthcare. IT proposes many beneficial attributes in easing the documentation processes in healthcare but also creates a host of challenges. Healthcare Information and Management Systems Society (HIMSS) conducted a survey in April 2009 on the population of 432 nurses and IT workers. The information is determined on a scale of 1 to 7 , where one is the lowest score and seven is the highest. Usability is one problem that is noted with bedside computer charting that takes the nurse's attention away from the direct patient care time. The HIMSS survey illustrated on this 1 to 7 scale, a result of 4.2 where IT staff felt that too often they had to chart the same information in different places [1]. The challenge created is that documentation for the healthcare professional at the patient's bedside becomes straightforward and consistent in a user-friendly manner.

Another challenge is that healthcare technology lacks connectivity that creates charting difficulties for healthcare staff. The term connectivity means the ability to move from screen to screen in a time efficient manner while satisfying patient care needs and addressing appropriate information without needless repetition of information in a timely manner

Manuscript received June 2, 2012; revised July 5, 2012.

E. Nwosisi is with the Kaplan University and Northern Westchester Hospital, Mount Kisco, NY (e-mail: enwosisi@msn.com).

L. Carl is with the Kaplan University School of Nursing, Chicago, Illinois (e-mail: 1carl@kaplan.edu).

C. Nwosisi is with the Computer Science Department, The College of Westchester, White Plains, NY USA and Department of Thoracic and Cardiovascular Surgery, Montefiore Medical Center, Bronx, NY USA (e-mail: cnwosisi@cw.edu).
[1]. The problem is that internally, nurses are talking to computers at the bedside instead of to patients. Too many computer clicks are needed to navigate through to an intervention, in a timely manner, forcing patients to wait for care. Fig. 1.

An intervention has redundant informational seeking fundamentals that reduce usability. Some have lengthy linear connectivity processes that create distractions for healthcare workers to navigate through the electronic medical record (EMR). Rather than to concentrate on the patient, the problem is that bedside charting takes away from the direct patient care time due to healthcare information technology lacking connectivity and usability [1]. The problem is that IT connectivity and usability could exist in healthcare simultaneously with efficient patient-centered quality care.

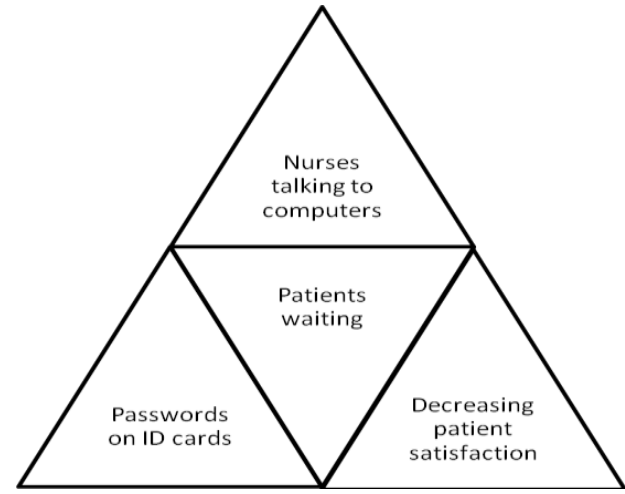

Fig. 1. Healthcare IT problems [13]

The problem addressed in this proposal in is that IT documentation does not always provide precision upon reviewing the information. For example, a system that is not specialty specific may not ask the accurate questions to get the pertinent information for optimal patient care. Also considered is duplication of information that may generate elimination of vital data. Research has shown that healthcare workers is comfortable with the paper chart and may be resistant to IT systems [2], [3]. With a survey score of 3.83 , healthcare workers tend to feel that nurses do not play an important enough role in the attainment of IT connectivity and usability applications [1].

The goal of the problem is investigated, as the research justly acknowledges that IT connectivity and usability is lacking. The specific research question posed for this proposal is noting the background of the problem. In a patient centered environment a multitude of unnecessary IT steps in addition to the lack of ease in navigating the IT system is very time-consuming, resulting in the diminution of valued hands-on care time being taken away from patient care. 
Shaping any relationship between the lack of connectivity and usability, entailing too many steps that produce errors in IT documentation and the increase for distractions in bedside patient care involve reducing lengthy and timely documentation processes that cause delays in efficient patient care management [4], [5], Fig. 2.

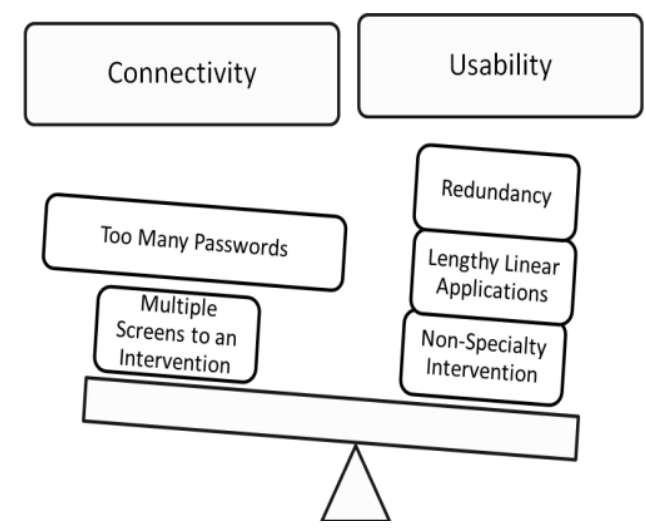

Fig. 2. Problem identification [13], [14]

The purpose of this research study is to analyze IT connectivity and usability using qualitative meta-analysis.

The meta-analysis is organized as follows: Section 2 provides details of the Theoretical Framework/ Conceptual model, Section 3 discusses the Implementation and Change and Section 4 presents the conclusion and suggestions for future work.

\section{THEORETICAL FRAMEWORK/CONCEPTUAL MODEL}

Contributions researching any relationship between connectivity and usability of informational technology in delivering efficient patient centered care require the use of a conceptual framework to underpin the theory that optimal patient care is provided using computer documentation. Nursing theories create a model of critical thinking so analysis of the literature is standardize across the literature review. The person, nursing, environment and health are the four component parts of the theoretical foundation of the meta-analysis. [6]. Fig. 3.

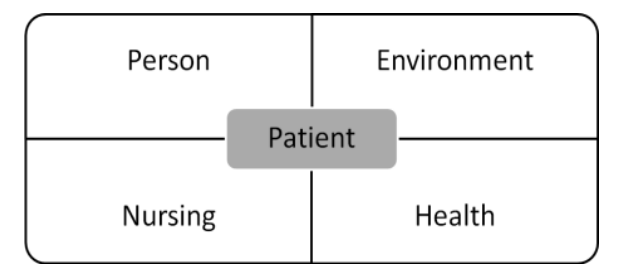

Fig. 3. Theoretical frameworks/conceptual model [6]

Starting with a middle-range theory of nursing's intellectual capital, the person, one considers that patient centeredness needs the nurse not the computer. The concept of concentrating on the patient creates an atmosphere that is conducive to the patient's well-being [7], [8]. Nursing theories have been used to mold research studies for many decades. For the purpose of the meta-analysis, Imogene's King theory will be used to mold and shape the research study. The act of caring is an important and vital attribute in the atmosphere that is to make up the environment surrounding the patient [9]. The link among environment, health, nursing, patient care, and computer documentation is the imperative right deserving of a person in the healthcare sector to have logical ease in IT documentation, in avoidance of having obstruction to healthcare.

When looking at nursing in relation to computer documentation, it is important to examine the patient to nurse as a caregiver relationship, the patient's ability to care for oneself, nursing knowledge and the conduciveness of the atmosphere to promote healing [7]. The difficulty in providing a caring environment that would be concentrated around the patient exists in trying to accommodate all aspects of healing and yet satisfy all patient comfort needs through computer documentation. This concept mirrors King's theory, which is labeled social systems where the interconnectedness of the patient and the nurse should not be confused by a lack of IT connectivity or usability [9].

The patient needs to exist in a caring and nurturing environment and for this flow to continue the nursing experience should be knowledgeable, current and capable of meeting patient care needs through computer documentation. For over a century, nursing has been trying to develop a complete nursing theory that encompasses the complete function of nursing care, theoretical functions and emotional needs connected to technology. King's model supports analysis of patient centeredness when evaluating IT connectivity and usability [9].

In looking at health, a new method of thinking emerges. In keeping the patient as the central focus, health becomes a totality of different thoughts. This may lead one to think that nursing theory has crossed over to the medium of parapsychology, however is not nursing, a sum of various sciences [6]? The examination of [10] itemization of its presence depicts the building blocks of health. Health should mirror the comprehensiveness of holistic care, patients' vulnerability, intimacy, sensitivity and the ability to adapt. The accomplishment of all these factors not only leads to optimal patient well-being but to a greater sense of quality thought processes.

The groundwork set here is the middle range grounded theory as defined by [6] to have at least one or more concrete images or ideas as part of the conceptual framework of the theory. One could contradict and say that the conceptual model is depicted here due to the combined delineation of references, in the manner of which this theory combined several theorists' ideas to formulate one on patient centeredness [6], [11]. However, the evolvement depicted in this study focuses on the intricacies of dealing and concentrating on the patient based issues with connectivity and usability that looms in the distance.

\section{IMPLEMENTATION AND CHANGE}

In order to improve healthcare delivery, the meta-analysis inspects informational technology in healthcare involving problems associated with lack of connectivity and usability within the documentation system and the relationship that evolves from direct high-quality patient care. IT in healthcare involves the converting of traditional patient-care paper chart to an electronic health record (EHR). The EHR incorporates different aspects of the patient healthcare chart electronically and allows the protected information to be accessible to 
healthcare workers even remotely. Nationally, the goal is to achieve a faster and more efficient method of charting and archiving patient information. By charting electronically, a plan facilitates the betterment in patient care [1]. The meta-analysis elucidates the implementation process of such a plan for easing the lack of connectivity and usability of the electronic health record with the goal attainment of efficient patient care [12].

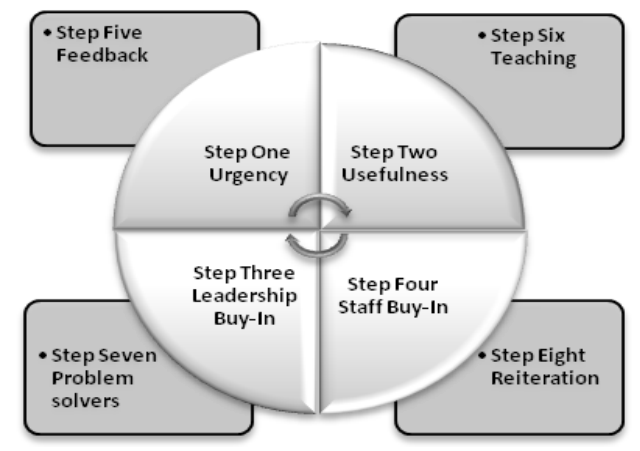

Fig. 4. Implementation [13].

The steps for the implementation of the plan are the Eight-Step Kotter's Change Model. The learning groups are divided by similar needs. Each group is divided into phases; phase one has a specific start time, the goal attainment time, a time for the evaluation of the plan and a re-evaluation era. Advisingly, [13] offers continuous promoting of the process that is to be utilized in implementing the plan, especially when new members are present. This move facilitates continuous updating of all members, ensuring that no one is amiss. Formulating order sets that guide nursing staff through specialty related protocols is the manner in which IT facilitates optimal patient care. When looking at cost, IT is not the only portion considered; one must look at business management and make decisions based on healthcare dealings as well [14]. Healthcare costs have been steadily increasing for decades [15]. Unfortunately, having the benefits as well as the costs of information technology only adds to healthcare costs. Moreover, the factor of expenditures has weight in deciphering through the perplexity [16].

Kotter's model is interjected to implement the plan, in order to facilitate the best possible improved outcome. Step one is the creation of urgency by illustrating decreasing patients' satisfaction survey scores based on patients' perception of their care [13]. Questions are asked that addresses patients' opinions in regards to nurses' attentiveness and friendliness, nurses willingness to help, nurses' call response time and patients' satisfaction that needs were met [17]. Demonstrating the opportunity to upgrade or revise current applications with additional diverse functions that streamlines information is highly suggested. One begins the proclamations as a means of advertisement of such a process. A recent smooth transition with improved results inspires organizations and their staff to encourage the support of subsequent progressive upgrades.

Step Two of a projected upgrade lends itself to numerous site visits, which assures organizations of the usefulness, the applicability and the projected results [13]. Thus, the more applications are universal, the easier the usability will be across the healthcare environment. Patients and their families are the center of the nurses' roles in any health care relationship. Patients depend on nurses to not only care for them but also to provide information, teach them about their disease processes and most of all teach health-maintenance discharge instructions. As one could see, a nurse's role is already multifaceted and time-consuming to meet the patient care needs. It is not to the patient's advantage to add information technology with connectivity and usability issues that would interfere with a nurse's or healthcare worker's busy role [18].

Linking electronic health records is another means of making IT connectivity and usability more conducive for nurses and other healthcare professionals. Therefore, a healthcare worker could access a comprehensive link and retrieve patient information without repeating questions to patients whose health and attentiveness may be compromised. A group sagely referred to as Super-users is identified in the process, as enthusiastic and IT literate facilitators that is put in place to facilitate training staff. Here is where the skeptics are removed; recognition and removing hindrances avoid delays in advancing the change [13], [14]. The latter probably is the most difficult to recognize and accomplish, especially without hurt feelings.

Implementation begins with the image of what is to be accomplished. Universal applications come in handy when, for example, a shared Obstetrical (OB) patient needs an evaluation. One may access that patient's file directly from the shared database without having to defer to other lengthy means of indirect communication of phone calls, lengthy paper file searches, conventional mail, radiology and laboratory inquiries [18], [19], [3].

Steps Three of the Kotter's 8-Step Change model produces an illustration that depicts the intended ease of acquiring the information in reduced and timely steps to facilitate the connectivity and usability aspects of the computer charting problems [13], [3]. Vision and mission statements exemplify these goals. Satisfying various tiers is necessary. Therefore, penetrating leadership, managerial, staff meetings and holding town meetings are ideal opportunities to reveal the proposed improvements.

Step Four expands on the preceding steps where the intended upgrade is not allowed to be forgotten. Reference to the upgrade should be excessive and open, as the need arises [13], [14]. Using a test mode, the organization should build applications that healthcare workers could access and "play with" to become familiar with the ease of the intended newer applications. Hands-on manipulation provides a more realistic view of what is to be expected. Shortening the steps of screen-to-screen manipulation is a desired goal.

In Step Five, one re-evaluates acceptance by obtaining feedback. The project and resources are assessed on a continual basis. Nurses have been known to be resistant to change and provisions should be made to calm fears [20]. Pay attention to suggestions and make adjustments as necessary. In particular, collaborate with the staff that performs direct patient care [13]. Again, this period is used to avoid obstacles and remove skeptics [20]. Job performances and description measures are assessed to ensure that the correct skill mix is utilized. Frequently, compensation is a necessary factor. In getting all committed, nursing management needs to be sensitive to doctors' reservations in the IT world and support their learning, but it does not end there. A concern has been 
raised of "the cookbook" sense of practicing medicine and the fear of physicians decreasing their independence in providing efficient patient care [21], [14]. The environment is to be such that is conducive to their learning in non-intimidating or condescending settings. The older doctors could be the most resistant [22]. Nurses as well as physicians should be part of the order sets-making processes.

Step Six of the Kotter's 8-Step Change model revolves around the change itself. Change must be effective with a strong united team-like approach with well-trained Super-users on board as well to assist those that fail to remember the processes [13]. Super-users are to be available for a full week 24/7, which makes use of the theoretical basis of getting all users comfortable with the implementation, similar to the moving stage of Lewin's theory [23], [13]. The Super-users are to be commended for their tasks in subduing unnecessary stress by being available to assist peers. Universal Go-Live are to be implemented in stages, by small increments of clinical areas, for example, medical-surgical, obstetrics and then pediatrics. Within a few days, the second phase is to be implemented and before long, the balance is implemented. Small steps are better than one large one [13]. Organizations need to follow the model that is supposed to result in a timely progression.

Step Seven regards a successful start, as only the beginning and one is not to stop the process at this point. For continuing successes, one is required to have systems in place to solve problems proactively. Assistance needs to have continued timely access [14], [5]. Documentation of the process is vital for future replication, if necessary. Ease in replication of the process determines success, so that one is able to recognize the positives and be mindful of the negatives as well [13]. Settling for what worked at present is to be avoided and one is always seeking other resources to support the change.

Step Eight of the Kotter's Change model is to remind the organization continually of the change and its' benefits. Senior leadership is to show appreciation of the change and be an exemplar for managers and staff. All participants in the course of action are to be commended publicly if possible for their contribution to the process [13].

Keep the processes alive. Discuss the change in the orientation process when new staff is hired. Keep new staff and leaders well informed of the information as staff uproot. One should never have to guess the origin of the change. Caution should be mentioned in having patience in the process, since that moving too fast could obliterate the advancement of the change. In addition, the organization needs to take into consideration budgeting for any necessary applications or personnel essential to propel the process forward.

Evaluating the impact of this proposed planned change from the community interests will be a bit indirect, because it is based on a subjective premise of the public. One manner in which to accomplish obtaining such information is to examine patient-care survey results [24]. Regrettably, some patient care surveys results are bias due to the size of the facility in question. For example, a small community hospital with less than 50 beds have a great chance of the healthcare staff being neighbors and friends; patients of such a facility could tend to give higher scores, as opposed to a patient of a 550-bed facility in a large urban city. One must weigh these considerations even when evaluating the results on a national level. The Press Ganey survey results are a national based tool that is used by many facilities nationwide [25]. The result of this tool may well be used to assess community interests.

The Hospital Consumer Assessment of Healthcare Providers and Systems (HCAHPS) survey is another tool that is used to identify community needs or interests of patients following recent hospitalizations, which again looks at patient scores, and is the method of evaluating patients' contentment [17]. Organizations may also use community focus groups and community interests' polls. Internationally, researchers have created their own tools to evaluate the success of IT implementation and as it happens, patient's feedback is recognized for the basis of functionality. In evaluating patient centered care at the bedside following the adoption of IT documentation in Austria, a homemade tool is utilized to assess patients' feedback [3].

Prior to embarking on any major project, it is advisable that an organization knows that they are satisfying their community's interests. If the intended plan does not meet the needs or the interests of the intended community then a potential financial loss ensues.

\section{CONCLUSION}

The meta-analysis of computer documentation and nursing care demonstrates that in the computer charting process, it is advantageous to identify the problem, explore sound theoretical framework to find a solution and start a plan to solve the issues that are identified. A review of the findings gathered from three libraries Cochran, Medline and Cumulative Index to Nursing and Allied Health Literature (CINAHL) results with the information that healthcare information technology is an important part of patient care. The plan is to integrate information technology in such a manner that computer charting remains patient care focused. In reviewing the research, implementation needs a solid evidence based method for releasing the desired effects across a population with techniques of evaluating acceptance and understanding the solutions. Fig. [6].

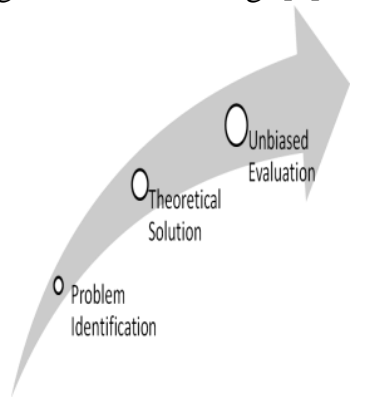

Fig. 5. Conclusion: Original work.

The meta-analysis used the Eighth Step Kotter's Plan for implementation [13]. The ultimate goal is to have patients feel that their needs are being met. Researchers have identified several ways of meeting this goal. One of them is direct feedback from the patients themselves [17], [9]. The national patient satisfaction survey is a system that is at best 
insures either the patient or the facility itself does not bias results. It takes the onus off both. Internationally, individual countries recognize the importance of patients' opinion. As an exemplar, an Austrian hospital created a patient's opinion tool to evaluate its' IT connectivity and usability issues [3]. Lengthy password mixed with numbers, case sensitivities and characters make for a more secure method in avoiding computer hacking but can hinder care at the bedside for the healthcare professional timely duties. Healthcare information is confidential and vigilance needs to be considered in the avoidance of unintentional disclosure of private patient information [14], [26].

In accordance with [13], [14], reliability regarding system failure and prompt maintenance encourages staff buy-in to the progression of IT documentation, which augments patient centered care. A recent smooth transition with improved results inspires organizations and their staff to encourage the support of subsequent progressive upgrades. As indicated by [15], [27], redundancy in documentation, lends to inconsistent connectivity prototype that leads to dissatisfaction in the healthcare worker. Vital signs are a common example of this process. It should be an easily accessible function but using different systems that do not see each other make attaining results an exasperating feat, especially remotely. A Universal system is a national incentive goal, according to [28]. However, linking systems already in place with new ones is a costly undertaking, especially when applying or updating software and hardware [14].

Though a universal system is ideal, systems would have to be comprehensive to envelop all aspects of healthcare. At present, many systems are generalized but some have the ability to have systems' upgrades, which enhance some specialties, while others are so specialty specific that multiple systems are needed for healthcare organizations to function [14]. With healthcare today being so technologically advanced and specialty specific, healthcare workers require an IT system that accommodates specialty criteria. In order to provide high-quality patient centered care, IT documentation needs to address the stakeholders' concerns, which in this case are the patients, so that timely illness-specific interventions are initiated [29]. Comprehensible and user-friendly IT usability and connectivity methods are critical in addressing patients' concerns thoroughly and opportunely, to increase patients' satisfaction consequently, according to [14].

For instance [30] positions, having a system that is noted to fulfill evidence based IT practices. The intervention screens are uncluttered and nonlinear with windows for mini dropdowns and fill-ins. Prompts to subsequent necessary fields are driven by national standards that decrease the chances of erroneous charting errors or omissions [29]. In addition, the system easily links office chart records from pre-hospitalization including laboratory results and follows the patient throughout all areas of the hospitalization. The entire system's charting mechanism meets national standards and the national organizations' guidelines for the specialties involved. Historical information entered populates identical fields in other areas, reducing the need for redundant documentation. An advantageous aspect of IT systems is the ease in connectivity in that one does not have to purchase additional computers, depending on the original memory size or servers, with the attainment of full contractual warranties. The information is archived with a secondary archive repository [30]. This move may well prove to be a financial advantage for the interested organization.

The clarity in documentation should exist well before it reaches the patient's bedside. Research efforts still need to be accomplished in decreasing connectivity and usability IT problems at the bedside and thus increase patient-centered care. According to [31], [32], healthcare workers' age and intellectual ability are to be considered in developing IT functions. For example, ease in correcting errors, location of buttons, ability to increase font as needed for older healthcare workers with declining vision and the ease of undoing errors are just few considerations for IT designers in developing and maintaining healthcare information systems, at the bedside for the perseverance of high quality patient centered care. Until IT documentation for healthcare staff at the patient's bedside is straightforward and consistent in a user-friendly manner, additional research is needed for patient centered care.

\section{ACKNOWLEDGMENT}

E. Nwosisi thanks her Dear husband and family for continued support and perseverance throughout the process. She also thank her parents, especially her mother Venice T. Lewis RN ACM whose endless enthusiasm, generosity, inspiration and compassion has propelled her to aspire excelling in life.

\section{REFERENCES}

[1] McKesson, (April 2, 2009). HIMSS 2009 informatics nurse impact survey. $\quad$ pp. 1-22. [Online]. Available: http://www.himss.org/content/files/HIMSS2009NursingInformaticsIm pactSurveyFullResults.pdf.

[2] J. J. Gooch, "Integration and prioritized data essential for EHRs," Managed Healthcare Executive, pp. 32-33, September 2008.

[3] T. Schiabetsberger, E. Ammenwerth, S. Andreatta, et al., "From a patent-based transmission of discharge summaries to electronic communication in healthcare regions," International Journal of Medication Informatics, vol. 75, pp. 209-215, 2006. [Online]. Available: http://www.lina-schwab.de/Publikationen/z36.pdf.

[4] C. J. Wallace, S. Bigelow, X. Xu, et al., "Collaborative practice usability of text-based, electronic patient care guidelines," CIN: Computers, Informatics, Nursing, vol. 25, pp. 39-44, 2007.

[5] A. M. Wichansky, "Usability testing in 2000 and beyond," Ergonomics, vol. 43, no. 7, pp. 998-1007, 2000.

[6] J. Fawcett, "Contemporary Nursing Knowledge: Analysis and evaluation of nursing models and theories," 2nd ed. Philadelphia, PA: F. A. Davis, 2005, ch. 5, pp. 88-127.

[7] B. McCormack and T. V. McCance, "Nursing theory and concept development or analysis: Development of a framework for person-centred nursing," The Authors. Journal Compilation, pp. 472-479, 2006. DOI: 10.1111/j.1365-2648.2006.04042.x.

[8] C. L. Covell, "The middle-range theory of nursing intellectual capital," Journal of Advanced Nursing, vol. 63, no. 1, pp. 94-103, 2008. DOI: 10.1111/j.1365-2648.2008.04626.x.

[9] B. K. Kileen and I. M. King, "Viewpoint: Use of king's conceptual system, nursing informatics and nursing classification systems for global communication," International Journal of Nursing Terminologies and Classifications, vol. 18, no. 2, pp. 51-57, May 2007.

[10] D. Finfgeld-Connett, "Qualitative convergence of three nursing concepts: Art of nursing, presence and caring," Journal of Advanced 
Nursing, vol. 63, no. 5, pp. 527-534, September 2008. DOI: 10.1111/j.1365-2648.2008.04622.x.

[11] R. Cader, S. Campbell, and D. Watson, "Cognitive continuum theory in nursing decision-making," Journal of Advanced Nursing, vol. 49, no. 4, pp. 397-405, February 2005.

[12] J. H. Frost, M. P. Massagli, P. Wicks, et al., "How the social web supports patient experimentation with a new therapy: The demand for patient-controlled and patient-centered informatics," in Proc American Medical Informatics Association Symposium, pp. 217-221, 2008.

[13] Mindtools, "Kotter's 8 step model for change," 2009. [Online]. Available: http://www.mindtools.com/pages/article/newPPM_82.htm.

[14] Proctor P. Reid, W. D. Compton, J. H. Grossman, and G. Fanjiang, Editors, "Information and communications systems: The backbone of the health care delivery system," in Building a Better Delivery System: A New Engineering/Healthcare Partnership. 2005, ch. 4, pp. 63-82, The National Academies Press, Washington, D.C. [Online]. Available: http://www.nap.edu/openbook.php?record_id=11378\&page $=63$

[15] G. L. Glandon, D. H. Smaltz, and D. J. Slovensky, "Austin and Boxerman's: Information systems for healthcare management," 7th ed. Chicago, IL.: Health Administration Press, 2008.

[16] J. J. Saleem, E. S. Patterson, L. Militello, et al., "Impact of clinical reminder redesign on learnability, efficiency, usability and workload for ambulatory clinic nurses," Journal of the American Medical Informatics Association, vol. 14, no. 5, pp. 632-640, June 2007. DOI 10. 1197 /jamia.M2163.

[17] S. B. Frampton, "Creating Patient Centered Care," The American Journal of Nursing, vol. 109, no. 3, pp. 30-33, March 2009.

[18] M. A. Spatz, "Personalized health information," The American Journal of Nursing, vol. 109, no. 4, pp. 70-72, April 2009.

[19] P. T. White, "Practitioner application," Journal of Health Management, vol. 51 , no. 1 , pp. 58-59, 2006.

[20] S. Timmons, "Nurses resisting information technology," Nursing Inquiry, vol. 10, no. 4, pp. 257-269, 2003.

[21] B. M. Averbeck, "Bringing evidence-based practices into practice," Health Management Technology, pp. 20-22, 2005.

[22] A. Yarbrough and T. Smith, "Technology acceptance among physicians: A new take on TAM," Medical Care Research and Review, vol. 64, pp. 650-672, 2007.

[23] T. Lee, "Nurses' perceptions of their documentation experiences in a computerized nursing care planning system," Journal of Clinical Nursing, vol. 15, no. 11, pp. 1376-1382, September 2006. DOI: 10.1111/j.1365-2702.2006.01480.

[24] R. Braden, S. Reichow, and M. A. Halm, "The use of the essential oil Lavandin to reduce pre-operative were to anxiety in surgical patients," Journal of PeriAnesthesia Nursing, vol. 24, no. 6, pp. 348-355, 2009.

[25] P. Ganey. Hospital checkup report: Physicians. The Press Gany Associated Incorporated, 1(3): 1-5. [Online]. Available: pressganey.com/galleries/default-file/physician-report.pdf

[26] R. Pak, M. M. Price, and J. Thatcher, "Age-sensitive design of online health information: Comparative usability study," Journal of Medical Internet Research, vol. 11, no. 4, 2009. DOI: 10. 2196/jmir.1220.

[27] C. Oroviogoicoechea, B. Elliott, and R. Watson, "Review: Evaluating information systems in nursing," Journal of Clinical Nursing, vol. 17, no. 5, pp. 567-575, 2008. DOI: $10.1111 /$ j.1365-2702.2007.01985.x;

[28] J. Halamka, M. Aranow, C. Ascenzo, et al., "Health care IT collaboration in Massachusetts: The experience of creating regional connectivity," Journal of the American Medical Informatics Association, vol. 13, no. 3, pp. 596-601, 2006. DOI 10.1197/jamia.M1866.

[29] K. B. Eden, R. Messina, H. Li, et al., "Examining the value of electronic health records on labor and delivery," The American Journal of Obstetrics and Gynecology, vol. 199, no. 3, pp. 307-309, 2008.

[30] Obix, Obix Perinatal Data System, 2009. [Online]. Available: http://www.obix.net

[31] R. G. Adams and P. Langdon, "Principles and concepts for information and communication technology design," Journal of Visual Impairment and Blindness, vol. 97, no. 10, pp. 602-611, 2003. ISSN 0145-482X.

[32] R. J. Koubek, D. Benysh, M. Bucks, et al., "The development of a theoretical framework and design to a process usability assessment," Ergonomic, vol. 46, no. 1-3, pp. 220-241, 2003. DOI: 10. One 080/001401. 3021000035244 .
E. Nwosisi has been in nursing for 29 years. She has acquired national certification in Inpatient Obstetrical Nursing, Electronic Fetal Monitoring and Obstetrical Ultrasound. Ms. Nwosisi is presently is a member of and an instructor for her national specialty organization, the Association of Women's Health and Neonatal Nursing (AWHONN). Ms. Nwosisi obtained her Master's of Science degree in nursing with a concentration in Nursing Administration from Kaplan University, Chicago, Illinois 2010.

She started at New York University Hospital (NYUH) after obtaining a Bachelor of Science degree in nursing from Herbert H. Lehman College of the City University of New York, 1983. At NYU, she worked as a Senior Nurse Clinician and had the benefit of the preceptor role, as well. In 1990, Ms. Nwosisi joined the Northern Westchester Hospital (NWH) community of Mt. Kisco, NY, where she quickly became a preceptor there, as well. Her experience spanned specialty nursing, in Oncology, Pediatrics, Medical/Surgical and Maternal Child Health, prior to choosing a permanent position on Maternal Child Health in 1991. She has been diligent in researching patient centeredness and information technology, resulting in her presenting in Shenzhen, China in 2012.

Ms. Nwosisi BS MS RNC currently possesses NWH Clinical Ladder Level V status. She is privileged to be a member of the Nursing International Honor Society and National Honor Society Alpha Beta Kappa. Additionally, she is a member of the National Honor Society of Nursing Sigma Theta Tau International for her leadership skills and lifetime achievements in nursing. She is consulted habitually in Electronic Fetal Monitoring and is an obstetrical resource for electronic IT charting for Leadership and the Medical Information System department at NWH.

Dr. Linda Carl is a graduate nursing professor and Chair of the Faculty Development Committee at Kaplan University, Chicago, Illinois, USA. Dr Carl was born in Washington, DC, and earned a doctoral degree in educational leadership practice from the University of Phoenix, Phoenix, Arizona, a Master degree in nursing administration from George Mason University, Fairfax, Virginia, a bachelor degree in nursing from The Catholic University of America, Washington, DC, and a bachelor degree in general studies with a focus on psychology from the American University, Washington, DC.

She has taught formally online and on ground in US higher education for the past ten years. She has published in hard copy and online in the areas of education, health reform, public policy, critical care, emergency nursing, performance improvement, trauma center criteria and systems of healthcare, caring for critically ill children, and risk management. She has presented at national and international conferences and before dignitaries and healthcare professionals worldwide.

Dr Carl has conducted education consultations in Spain, Portugal, Russia, Hungary, Australia, Hong Kong, Japan, England and France. Dr Carl's research focus is on innovative education system models and processes to support student, faculty, program and organizational success. Dr. Carl has performed peer reviews for Journal Critical Care, Public Health Nursing Journal and Merion publishers. Dr. Carl is a member of Sigma Theta Tau International nursing honor society.

Dr. C. Nwosisi obtained his Doctorate Degree in Computing from Pace University, Master of Science in Management of Technology from Polytechnic University and BA in Computer Science from Hunter College of the City University of New York. He has co-authored several papers in Scientific Journals and International conferences.

Currently, he works for Montefiore Medical Center in the Bronx, New York, USA and as an Adjunct Professor and Chairperson of the Technology Department at the College of Westchester in White Plains, New York, USA

For his contributions, Dr. Nwosisi received several recognitions. In 2010 and 2012 respectively, he received the Shining Star award in teaching from The College of Westchester. In 2010, he received the Upsilon Pi Epsilon Honors award for the Computing and Information Disciplines from Pace University. In 2007, he received the IEEE senior membership award. In 1993 and 1994 respectively, he was the recipient of the Recognition and Appreciation Awards from the Association for System Management. He has served as Program Chair and Conference Session Chair for various International Conferences and as a peer reviewer for the International Journal for Information and Education Technology. His current research interests include Decision trees, Machine Learning, Data mining and Pattern recognition. 